\title{
Diagnosis and management of fetal growth retardation
}

\author{
D James
}

What is fetal growth retardation?

Fetal growth retardation is best defined as the failure of a fetus to achieve its growth potential. ${ }^{1}$ Though this is the theoretically correct definition, in practice various less precise criteria have been used, including: measures of absolute size such as low birth weight (less than $2500 \mathrm{~g}$ ), and very low birth weight (less than $1500 \mathrm{~g}$ ); and measures of relative size (small for gestational age), variably defined as less than the third centile, less than the tenth centile, and less than two SD below the population mean for gestational age and sex.

These practical criteria have limitations, and comparison of studies using different definitions of fetal growth retardation is difficult. Because normal fetal growth is under intrinsic and extrinsic control by, for example, genetic, endocrinological, and nutritional factors, there is considerable variation in the incidence of pathological fetal growth depending on the population studied. ${ }^{2}$ In addition, not all 'small' babies by these definitions are pathologically grown, and conversely, some babies that are 'not small' may not have achieved normal pathological growth. Finally, the confounding effects of preterm birth are not easy to disentangle.

There is conflicting evidence as to whether the fetus that is small for gestational age has a higher mortality than the fetus that is appropriately grown when one allows for confounding variables such as congenital abnormality, intrauterine viral infection, and gestational age. ${ }^{3}$ The association between fetal growth retardation and mortality is not yet established and the question will only be resolved if sufficiently large numbers are analysed to take account of these variables.

Clearly there will be increased morbidity in fetuses that are small for gestational age with either a congenital anomaly or an intrauterine viral infection, the exact prognosis depending on the diagnosis. Even when these problems are excluded, however, in contrast to the mortality data, the fetuses that are small for gestational age do seem to be at increased risk of both short and long term morbidity. ${ }^{34}$ When one allows for confounding variables such as gestational age, race, gender, and socioeconomic group, there may be as much as six times the risk of neurodevelopmental handicap at one year of age in these infants compared with these infants who have grown appropriately. ${ }^{3}$ In addition, the effects may vary with the severity, ${ }^{4}$ the duration, ${ }^{5}$ and the type of fetal growth retardation, namely the degree of retardation of fetal head growth. ${ }^{6}$

\section{Which fetus is at risk of developing growth retardation?}

A number of epidemiological factors are associated with an increased risk of a fetus being small for gestational age. These include fetal sex, multiple pregnancy, birth order, socioeconomic group, maternal height and weight, ethnic group, and interpregnancy interval. ${ }^{7}$ Several authors have used these associations to try and predict the risk of the subsequent occurrence of a fetus that is small for gestational age. For example, Galbraith et al in a study of 8030 babies, ${ }^{8}$ claimed a positive predictive value of their risk scoring method of $9 \cdot 8 \%$ and a false negative rate of $2 \cdot 3 \%$ (sensitivity $69 \cdot 1 \%$, specificity $67 \cdot 1 \%$ ). Such risk scores have not been widely implemented; they are cumbersome to use, they have a poor predictive value, and they do not always predict the fetus that is pathologically small for gestational age.

A more practical approach is to identify pregnancies with specific risk factors for a fetus being small for gestational age compared with the normal population, and then to measure the growth of those fetuses serially with ultrasound to identify those that actually develop pathological growth. The following are examples of such risk factors.

Severe maternal malnutrition resulting in reduced energy intake and pathological fetal growth is seen during major famines, but is only likely to be encountered in developed countries if the mother develops a rare condition such as pancreatitis, active inflammatory bowel disease, or an eating disorder, or has a bowel resection. In such cases restricting the mother's dietary intake to less than $3.77 \mathrm{MJ} /$ day for six months will result, on average, in a fetus that is $240 \mathrm{~g}$ lighter than the fetus whose mother ate normally. ${ }^{9}$

Alcohol consumption in pregnancy is a cause of fetal growth retardation. For example, the odds ratio for a fetus being small for gestational age if a mother takes two drinks a day is 1.62 ( $95 \%$ confidence interval (CI) $1 \cdot 26$ to $2 \cdot 09$ ). This risk rises progressively with the number of drinks consumed. ${ }^{7}$ Mothers addicted to opiates have five times the risk of fetal growth retardation compared with controls. ${ }^{10}$

Essential hypertension alone is not associated with a greater likelihood of a fetus being small for gestational age, though this is contested by some authors. ${ }^{9}$ The risk of a fetus being small for gestational age is doubled if the mother has proteinuric pre-eclampsia alone, and quadrupled if this is superimposed on pre-existing hypertension. A mother with cyanotic cardiac disease has a risk of a fetus being small for gesta- 
tional age that is ten times that in the normal population. In chronic maternal renal disease the risk is related to the degree of renal dysfunction and hypertension. ${ }^{10}$ Sickle cell disease (and its variants), and systemic lupus erythematosus, are two other important diseases that are associated with a greater likelihood of fetal growth retardation. 9 10

A raised maternal serum $\alpha$ fetoprotein concentration in the first half of pregnancy, not associated with fetal abnormality, carries an increased risk of a fetus becoming small for gestational age later in the pregnancy. ${ }^{11}$ Other biochemical tests, largely discarded over the past 15 years, perhaps warrant further examination as predictors of fetal growth failure before it becomes clinically or ultrasonically apparent. Assays of human placental lactogen, oestriol, and Schwangerschaftsprotein-1 all have a sensitivity and positive predictive value for a fetus being small for gestational age of between $30-50 \% .^{12}$

The ability of Doppler ultrasound to predict the fetus at risk of growth retardation has been studied using recordings from both the uteroplacental and fetal circulations. In an early pilot study of uteroplacental waveforms in 126 pregnancies at 16-20 weeks, an abnormal waveform predicted $68 \%$ of pregnancies with fetal growth retardation with a specificity of $65 \%$, positive predictive value of $26 \%$, and a negative predictive value of $92 \% .{ }^{13}$ These encouraging results need to be confirmed by larger studies. Umbilical artery Doppler recordings later in pregnancy are relatively poor predictors of a fetus that is small for gestational age, though they do seem to be better at predicting subsequent fetal asphyxia. $^{14}$

Finally, maternal smoking is one of the most common contributing factors to fetal growth retardation (up to $40 \%$ ) in developed countries. ${ }^{9}$ The babies born to mothers who smoke cigarettes in pregnancy are approximately $250 \mathrm{~g}$ lighter than those born to mothers who do not smoke. ${ }^{715}$ Ironically the habit is too widespread to make it a practical proposition to carry out serial ultrasonic growth scans on all fetuses of mothers who smoke and furthermore, by itself it is a poor predictor of a fetus being small for gestational age.

Can we prevent fetal growth retardation? Giving up smoking during the first half of pregnancy, and stopping or reducing the drinking of alcohol during pregnancy both improve fetal growth. ${ }^{10}$ In areas of famine where severe maternal malnutrition results in fetal growth retardation, the effect can be prevented by giving the mother more to eat. ${ }^{9}$ It has been suggested, though not universally agreed, that conversion of opiate addicts to low dose methadone for the duration of pregnancy results in delivery of infants with weights comparable with those of non-addicted women of similar socioeconomic class. ${ }^{15}$

A patient who has hypertension before the onset of pregnancy should have the hypertension controlled. The evidence suggests that control of blood pressure before pregnancy reduces the risk of having a fetus that is small for gesta- tional age in the subsequent pregnancy, whereas treatment of hypertension during pregnancy has no appreciable effect on fetal growth. ${ }^{16}$ The high risk of fetal growth retardation if the mother has cyanotic heart disease is roughly halved if the patient has corrective surgery before pregnancy. ${ }^{9}$ For the patient with chronic renal disease, control of hypertension before pregnancy would seem to be of benefit, the most remarkable reduction of risk, however, is achieved by renal transplantation provided that proteinuria and hypertension do not continue after transplantation and that there is no evidence of graft rejection and renal function is normal. ${ }^{17}$ The result is also dependent on the patient taking small doses of immunosuppressive drugs such as prednisolone and azathioprine. Cyclosporin, on the other hand, seems to be associated with an increased risk of a fetus that is small for gestational age. In patients with sickle cell disease there is no information about whether prevention of recurrent crises by adequate hydration and prompt treatment of infection reduces the likelihood of a fetus that is small for gestational age. ${ }^{15}$

Though there is good theoretical and experimental evidence that bed rest should improve the uteroplacental blood supply and thus reduce the risk of fetal growth retardation, there is no convincing evidence of its value in practice. ${ }^{15}$

Treatment with low doses of aspirin, in contrast, holds out more hope for prophylaxis of fetal growth retardation. There have now been several reports that such treatment has resulted in a lowering of the incidence of pre-eclampsia, a fetus that is small for gestational age, or both, in at risk pregnancies. ${ }^{18} 19$ The results of the Medical Research Council (MRC) multicentre trial of treatment with low doses of aspirin are awaited with interest.

\section{Can we identify the fetus with growth retardation?}

Clinical inspection and palpation have sensitivity of $44 \%$, a specificity of $88 \%$, and a positive predictive value of $29 \%$ in predicting the fetus that is small for gestational age. ${ }^{20}$ Measurement of fundal-symphysial length is only slightly better with a sensitivity between $62-86 \%$, a specificity between $79-89 \%$, and a positive predictive value between $18-79 \% .{ }^{20} 21$ Though clinical measures tend to be unreliable screening tests, however, the more accurate results have come from studies of high risk pregnancies. ${ }^{21}$

Fetal ultrasonographic measurement remains the best method by which fetal growth retardation is identified. The most commonly measured fetal dimensions are biparietal diameter, head circumference, abdominal circumference, and length of femur. They are used either separately or together. All studies show that the abdominal circumference is the single most effective measurement for monitoring fetal growth and predicting fetal size, as it is reduced in both symmetrical and asymmetrical types of fetal growth retardation. Head and femur measurements are often unaffected in asymmetrical fetal growth retardation. ${ }^{22}$ Though serial fetal measurements and the plotting of growth trajec- 
tories (using charts appropriate for a given population) are the best methods of diagnosing pathological fetal growth, single measurements are less helpful. ${ }^{21}$ Prediction of fetal weight from a single measurement of abdominal circumference is associated with an error of about $16 \%$. ${ }^{22}$ This error may be greater at the extremes of size with a tendency to overestimate the size of small babies and to underestimate the size of big babies. ${ }^{21}$ Formulas for predicting fetal weight that incorporate abdominal circumference plus one or more other measurements reduce the error of the predictions. ${ }^{22}$

Serial ultrasound recording of fetal growth is possible in a small number of high risk pregnancies, but it is not feasible as a screening procedure for women who are not obviously at risk. A number of studies have been undertaken to assess the effectiveness of ultrasound measurements on one or at most two occasions in pregnancy to screen for the fetus that is small for gestational age in normal and low risk populations. The results are difficult to compare because of variables such as gestational age at the time of the scan, the prevalence of fetuses that are small for gestational age in the study population, the definition of small for gestational age, the measurements made, the skill of the observers, and the equipment used. The sensitivity varies from $43-94 \%$, the specificity from $84-92 \%$, and the positive predictive value from $20-70 \%$. ${ }^{20}$ These figures are not dissimilar from the ranges quoted for measurements of fundalsymphysial length. Indeed, in a comparative study in the same group of women, there was no difference between a single measurement of abdominal circumference in the last trimester and serial fundal measurements of length, though combining the two improved the accuracy. ${ }^{22}$

Of perhaps greater relevance is whether routine clinical or ultrasound screening for fetuses that are small for gestational age in the normal population is of any value in terms of outcome. For example, there have been five randomised controlled trials of ultrasonographic screening; only one of the five reported an improved outcome, the other four failing to show any benefit. ${ }^{20}$

\section{What should we do when fetal growth retardation is present?}

The first requirement is careful counselling. It is clear that there are limitations in our ability to identify the fetus that is small for gestational age accurately, and it is often impossible to distinguish the fetus that is pathologically growing from the fetus that is constitutionally or genetically small. A balance must be maintained in what we say to avoid misleading and confusing the mother therefore, so that we do not cause unnecessary anxiety, but succeed in conveying concern where appropriate.

When pathological growth is suspected, further evaluation is necessary both to confirm the diagnosis and to establish the aetiology. The first requirement is to confirm that the fetus is normal. Though every routine scan should include such an assessment, it is especially important if there is the possibility of fetal growth retardation. ${ }^{23}$ Further ultrasonic documentation of growth may be helpful in distinguishing the genetically or constitutionally small fetus (whose growth should follow the normal centile trajectories) from the pathologically growing fetus (whose growth, especially of the abdomen will fall away from expected trajectories). ${ }^{23}$ Because of the errors associated with ultrasonographic measurements, it is probably wise to ensure that the interval between growth scans is not less than two weeks. Invasive procedures have been used to evaluate the causes of growth retardation. Amniocentesis is of limited value, but placental biopsy (for rapid karyotyping) and umbilical cordocentesis (for karyotyping and viral serology) are being increasingly done. ${ }^{1522}$ These procedures are not without risk, however, and there are no clear guidelines as to when they are appropriate.

Specific management depends upon the cause. In the case of fetal abnormality this will be determined by the diagnosis and prognosis, the gestational age, and the parents' wishes. In the case of viral infection the prognosis is not always easy to predict, which makes counselling more difficult because it is difficult to detect which organs are affected in utero, and though the baby may be normal (though small) at birth, subtle damage (for example, hearing loss or moderate mental retardation) may not be identified until later in infancy. ${ }^{24}$

In other cases, such as uteroplacental vascular disease, recurrent bleeding, or idiopathic growth retardation, in which the baby is normal and there is no viral disease, the current approach to management can be considered under four headings.

\section{GENERAL MEASURES}

Giving up smoking and alcohol in the first half of pregnancy have both been shown to result in improved fetal growth, compared with those mothers who continue with these habits. ${ }^{10}$ As discussed above, despite good theoretical reasons the value of bed rest in reducing the risk of fetal growth retardation remains to be proved. ${ }^{15}$

ASSESSMENT OF FETAL WELLBEING

Apart from confirming the diagnosis of fetal growth retardation, serial ultrasound measurements of fetal growth give an indication of its severity and may indicate when fetal growth ceases. ${ }^{23}$ Over the last decade evaluation of fetal health had largely been undertaken by biophysical methods, which have thus replaced biochemical methods. ${ }^{12}$ Most of the biophysical methods were developed after retrospective evaluation of their ability to predict fetal asphyxia or death. They were introduced with great enthusiasm, yet most have not been subjected to prospectively controlled clinical trials. They are generally better at confirming normality (high specificity and negative predictive value) than in identifying abnormality (low sensitivity and positive predictive value). ${ }^{21}$

The importance of maternal perception of fetal movements has been recognised for a long 
time, and has been used in various methods of assessing fetal wellbeing. The value of 'kick charts' has recently been questioned by a study of nearly 70000 pregnancies in which the use of the charts was not associated with any lowering of the rate of fetal loss. ${ }^{25}$ Whether they would produce better results in a high risk group has not been assessed.

Measurement of the fetal heart rate at rest is currently the most widely used method of fetal assessment. Its value in predicting fetal hypoxia was well reviewed by Devoe et al. ${ }^{26}$ Using various outcome measurements of fetal hypoxia, the measurement of heart rate at rest had a sensitivity of $50 \%$, a specificity of $90 \%$, a positive predictive value of $40 \%$, and a negative predictive value of $95 \%$.

The fetal biophysical profile score has two underlying principles: firstly, that the fetus subjected to chronic asphyxia will in time undergo changes as a result of the effects of the hypoxia on renal function (amniotic fluid volume) and cerebral function (fetal tone, movements, breathing, and variability of heart rate); secondly, by combining the five measurements in one assessment a more accurate prediction of fetal risk can be obtained than from any one measurement alone. The overall gross perinatal mortality rate in high risk monitored patients is about 7:1000 (corrected for lethal abnormalities and rhesus disease it is below 2:1000) compared with an untested population figure of $14: 1000 .^{27}$ The biophysical profile score gives higher incidence of normal results $(97 \cdot 5 \%)$ than measurement of the fetal heart rate at rest $(80 \%)$ and a lower false negative rate (the risk of a fetal death occurring within one week of a normal result from the biophysical profile score is $0 \cdot 68 / 1000$ tests compared with $4 / 1000$ for measurement of fetal heart rate). ${ }^{27}$

Doppler recordings from fetal vessels (umbilical artery, descending aorta, and cranial arteries) seem to be sensitive indicators of the fetal circulatory state. The absence of end diastolic recordings in the umbilical artery, or descending aorta, or both, in growth retarded fetuses seem to be associated with a high incidence of fetal hypoxaemia and the subsequent development of perinatal asphyxia. ${ }^{14}$ The clinical management appropriate for growth retarded fetuses with abnormal blood flow, however, has to be established in prospective randomised studies before any recommendations for general clinical use can be made.

Cordocentesis can be used to measure fetal blood gas tensions and it is attractive to consider this to generate a rational approach to the management of growth retarded fetuses. Though this approach has been strongly advocated by some authors, ${ }^{1522}$ there are no prospective data linking fetal cord blood gas values to outcome, either in the short or the long term. What are the indications for cordocentesis? If the tensions are normal, when should the procedure be repeated? Furthermore, cordocentesis is itself a potentially hazardous procedure. Until the outcome data from prospectively conducted intervention trials have been produced, cordocentesis for fetal blood gas analysis should not be introduced into clinical practice.

\section{SPECIFIC TREATMENTS}

Aspirin has already been mentioned as possibly preventing fetal growth retardation. There is also some evidence that it may limit or improve fetal growth retardation once present. Trudinger et al showed in a double blind placebo controlled study that women with high umbilical arterial velocities given $150 \mathrm{mg}$ aspirin daily delivered babies over $500 \mathrm{~g}$ heavier than the control group. ${ }^{19}$ In that study, however, though most of the women were recruited because their fetuses were small for gestational age, there were no details of fetal size at entry.

$\beta$-Sympathomimetics increase fetal birth weight in animals primarily by improving uterine blood flow. There is, however, no clear evidence of their value in human growth retardation.

Solcoseryl is a haemodialysate containing no protein or antigens derived from calf blood. Theoretically it may improve fetal growth by effects both on fetal metabolism and on the maternal circulation. A small study of its use in pregnancies complicated by fetal growth retardation reported a tenfold lower incidence of babies that were small for gestational age in the treated group. ${ }^{28}$ Allylestrenol is a synthetic progestogen that has been used in the management of both threatened abortion and of fetal growth retardation. It may improve the maternal uteroplacental circulation either by $\alpha$ receptor blockade or by antiplatelet action. More study is required of its value in fetal growth failure. ${ }^{15}$

The treatment of fetal growth retardation by nutrient supplementation (carbohydrate, lipids and amino acids, given both singly and in combination either to the mother or to the fetus) has been studied mainly in animals with experimentally induced growth failure, and with varying success. There are few data about humans. ${ }^{15}$

Maternal hyperoxygenation is another possible treatment for severe fetal growth retardation that requires further evaluation. In a preliminary study of 19 fetuses with severe growth retardation and abnormal umbilical artery Doppler measurements and fetal hypoxaemia, humidified oxygen (55\%) was given to the mothers for 24 hours a day. In four cases there was a rise in the calculated fetal thoracic aorta velocity for several days or weeks, and all four infants survived. In six cases there was no improvement in aortic velocity; among the six cases, there was one fetal death and four neonatal deaths. In the remaining nine cases initial improvement in aortic velocity was followed by a fall; there was one fetal death, four neonatal deaths, and four infants survived the neonatal period. ${ }^{15} 29$ Though these preliminary data suggest that maternal hyperoxygenation may improve fetal oxygenation and delay delivery until the fetus may survive outside the uterus, the benefit has not yet been proved, and the risks to the mother will have to be carefully monitored.

\section{DELIVERY}

The timing and method of delivery of the fetus with growth retardation are influenced by the factors discussed above. It may be relatively 
straightforward, for example, when the fetus has a lethal abnormality. When the fetus is normal, however, the decisions are not as easy, mainly because the necessary prospective randomised controlled studies with long term follow up into childhood have not been undertaken. A number of unanswered questions remain, therefore. For example, the current practice is to deliver the fetus with growth retardation once the biophysical criteria become abnormal or 37 weeks' gestation is reached, whichever is sooner. But is the fetus with growth retardation but normal biophysical measurements better delivered before the measurements become abnormal, even though that may expose the baby to the complications of preterm delivery? What is the role of cordocentesis in resolving this? What are the criteria for allowing vaginal delivery to be attempted?

Undoubtedly when vaginal delivery is undertaken the fetus with growth retardation is at greater risk of intrapartum hypoxia. The current practice is to monitor the fetal heart rate continuously during labour, and if this becomes abnormal to assess fetal capillary $\mathrm{pH}$ and expedite delivery if it is less than $7 \cdot 2$. In addition, many clinicians consider the presence of meconium in the liquor as an ominous sign. In general, normal heart rate patterns and the absence of meconium are indicative of fetal wellbeing during labour. When intrapartum acidosis occurs in the pathologically grown fetus it is usually at a faster rate than in the normally growing baby. In such cases, when the fetal heart becomes abnormal it is usually characterised by variable or late decelerations. ${ }^{30}$ Fetal heart rate monitoring and $\mathrm{pH}$ testing are, however, far from perfect ways of assessing intrapartum fetal health. They are associated with a relatively low positive predictive value and sensitivity in predicting fetal asphyxia, in whatever way it is defined. ${ }^{30} 31$ There is a growing need for improved methods of continuously assessing the health of the fetus with growth retardation during labour.

\section{Conclusions}

The diagnosis and management of the fetus with growth retardation remain high priorities in obstetric care. Current practice is, however, far from ideal. There is a need for a universally agreed definition of fetal growth retardation. There are limitations in our ability to diagnose pathological fetal growth. Many methods of predicting, assessing, and treating the fetus with growth retardation have been proposed. The outcome, however, both in the short and long term, of properly constructed prospective studies should be awaited before such strategies are introduced into clinical practice.

1 Chiswick ML. Intrauterine growth retardation. $\mathrm{Br} \mathrm{Med} \mathcal{J}$ 985:291:845-8.
2 Brar HS, Rutherford SE. Classification of intrauterine growth retardation. Semin Perinatol 1988;12:2-10.

3 Teberg AJ, Walther FJ, Pena IC. Mortality, morbidity, and outcome of the small-for-gestational age infant. Semin Perinatol 1988;12:84-94.

4 Stewart A. Fetal growth: mortality and morbidity. In: Sharp F, Fraser RB, Milner RDG, eds. Fetal growth. London: Royal College of Obstetricians and Gynaecologists, 1989: 403-12.

5 Fawer C-L, Calame A. Assessment of neurodevelopmental outcome. In: Levene MI, Bennett MJ, Punt J, eds. Fetal and neonatal neurology and neurosurgery. Edinburgh: Churchill Livingstone, 1988:71-88.

6 Harvey D, Prince J, Bunton J, Parkinson C, Campbell S. Abilities of children who were small-for-gestational age babies. Pediatrics 1982;69:296-300.

7 Alberman ED. Fetal undergrowth: epidemiology. In: Sharp F, Fraser RB, Milner RDG, eds. Fetal growth. London: Royal College of Obstetricians and GynaecoloLondon: Royal Coll

8 Galbraith RS, Karchman EJ, Piercy WA. The clinical prediction of intrauterine growth retardation. Am $\mathcal{f}$ Obstet Gynecol 1979;133:281-7.

9 Carlson DE. Maternal diseases associated with intrauterine growth retardation. Semin Perinatol 1988;12:17-22.

10 James DK. Risk at the booking visit. In: James DK, Stirrat GM, eds. Pregnancy and risk. Chichester: John Wiley, 1988:45-80

11 Evans J, Stokes IM. Outcome of pregnancies associated with raised serum and normal amniotic fluid AFP concentrations. $B r$ Med $\mathcal{F}$ 1984;288:1494.

12 Chard T, Howell RJS. Detection of fetal growth retardation by biochemical tests. In: Sharp F, Fraser RB Milner RDG, eds. Fetal growth. London: Royal College of Obstetricians and Gynaecologists, 1989:263-72.

13 Campbell S, Pearce JMF, Hackett G, Cohen-Overbeek TE, Hernandez C. Qualitative assessment of uteroplacental blood flow: early screening test for high-risk pregnancies. blood flow: early screening test
Obstet Gynecol 1986;68:649-53.

14 Marsal K. Fetal and placental blood flow. In: Sharp F, Fraser RB, Milner RDG, eds. Fetal growth. London: Royal RB, Milner RDG, eds. Fetal growth. London: Royal College of Obstetricians and Gynaecologists, 1989:279-308.
Nicolaides KH, Economides DL, Thorpe-Beeston G. TreatNicolaides KH, Economides DL, Thorpe-Beeston G. Treat-
ment of fetal growth retardation. In: Sharp F, Fraser RB, Milner RDG, eds. Fetal growth. London: Royal College of Obstetricians and Gynaecologists, 1989:333-61.

6 Redman C. Hypertension in pregnancy. In: de Swiet M, ed. Medical disorders in obstetrics. Oxford, Blackwell Scientific: 1984:149-91.

17 de Swiet M. Pre-existing medical diseases. In: Chamberlain G, Lumley J, eds. Pregnancy care: a manual for practice. Chichester: John Wiley, 1986:69-111.

18 Wallenberg HCS, Dekker GA, Makowitz JW, Rotmans P. Low-dose aspirin prevents pregnancy-induced hypertension and pre-eclampsia in angiotensin-sensitive primision and pre-eclampsia in
gravidae. Lancet 1986;i:1-3.

19 Trudinger BJ, Cook CM, Thompson RS, Giles WB, Connelly A. Low-dose aspirin therapy improves fetal weight in A. Low-dose aspirin therapy improves fetal weight in
umbilical placental insufficiency. Am $\mathcal{f}$ Obstet Gynecol umbilical placenta

20 Villar J, Belizan JM. The evaluation of the methods used in the diagnosis of intrauterine growth retardation. Obstet Gynecol Surv 1983;41:187-99.

21 Whittle MJ. An overview of fetal monitoring. Clin Obstet Gynecol 1987;1:203-22.

22 Campbell $S$. The detection of intrauterine growth retardation. In: Sharp F, Fraser RB, Milner RDG, eds. Fetal growth. London: Royal College of Obstetricians and Gynaecologists, 1989:251-61.

23 McNay MB. Diagnostic ultrasonography. Clin Obstet Gynecol 1987;1:29-53.

24 Sever JL, Larsen JW, Grossman JH. Handbook of perinatal infections. Boston: Little, Brown and Company, 1979.

25 Grant A, Valentin I, Elbourne D, Alexander S. Routine formal fetal movement counting and risk of antepartum late death in normally formed singletons. Lancet 1989;ii:345-9.

26 Devoe LD, Castillo RA, Sherline DM. The non-stress test as a diagnostic test - a critical appraisal. Am $\mathcal{f}$ Obstet Gynecol 1985; 152:1047-53.

27 Manning FA, Manticoglou S, Harman CR, Morrison I, Lange IR. Antepartum fetal risk assessment: the role of the fetal biophysical profile score. Clin Obstet Gynecol 1987; 1:55-72.

28 Kaplinski AK, Kurjak A. The treatment of growth retarded fetuses with haemodialysate (Solcoseryl): ultrasonic control. In: Kurjak A, Kossokk G, eds. Recent advances in ultrasound diagnosis. Vol 4. Edinburgh: Churchill Livingstone, 1984:174.

29 Nicolaides KH, Campbell S, Bradley RJ, Bilardo CM, Soothill PW, Gibb D. Maternal oxygen therapy for intrauterine growth retardation. Lancet 1987;i:942-5.

30 Steer PJ. Intrapartum monitoring in IUGR. In: Sharp F, Fraser RB, Milner RDG, eds. Fetal growth. London: Roya College of Obstetricians and Gynaecologists, 1989:381-7. 31 Steer PJ. Risks of labour. In: James DK, Stirrat GM, eds.
Pregnancy and risk. Chichester: John Wiley, 1988:105-35. 